BONPLANDIA 20(2). 2011

ISSN: 0524-0476

\title{
LA ETNOBOTÁNICA MÉDICA DEL ÁREA DE TRANSICIÓN PAMPEANO CUYANA
}

WALTER A. MUIÑO1

\begin{abstract}
Summary: Muiño, W. A. 2011. Medical ethnobotany of the transitional area between Pampa and Cuyo region. Bonplandia 20(2): 353-369.

In this study 85 plant species involved in home medicine of a rural community are recorded. This community is stated in NW La Pampa (Argentina) in the transitional area between Pampa and Cuyo region. Etiological and therapeutical interpretations of the diseases are studied by means of open and semi-structured interviewees made with male and female adults. Social actors who perform the skill of healing as soon as the present state of the traditional botanical knowledge regarding to home medicine are described too. The greatest diversity of uses belongs to the most abundant species and they are related to respiratory, digestive, urinary and orthopedic diseases. Processes of erosion related to the Hippocratic model of the traditional medicine become evident in contrast to other rural communities of South America as well as in supernatural therapies where religious symbols and rituals take part.
\end{abstract}

Key words: Ethnobotany, traditional medicine, medicinal plants, traditional botanical knowledge, Monte, Argentina.

Resumen: Muiño, W. A. 2011. La etnobotánica médica del área de transición pampeano cuyana. Bonplandia 20(2): 353-369.

En este estudio se presentan 85 especies vegetales que intervienen en la medicina local de una comunidad rural del NW de La Pampa, Argentina, ubicada en el área de transición entre la región pampeana y cuyana. A lo largo de entrevistas abiertas y semiestructuradas llevadas a cabo con adultos de ambos sexos, se analizan también las interpretaciones etiológicas y terapéuticas de las dolencias, y se describen a los actores sociales que desempeñan el arte de curar y el estado actual del conocimiento botánico tradicional en torno a la medicina local. La mayor diversidad de usos está en correspondencia con las especies más abundantes y se relacionan con trastornos de origen respiratorio digestivo, urinario y traumatológico. Se evidencian procesos de erosión en torno al modelo hipocrático de la medicina tradicional en contraste con otras comunidades rurales de Sudamérica así como en las terapias de carácter sobrenatural donde intervienen símbolos y rituales religiosos.

Palabras clave: Etnobotánica, medicina tradicional, plantas medicinales, conocimiento botánico tradicional, Monte, Argentina.

\section{Introducción}

La etnobotánica médica de los pueblos del mundo es un tema que hasta el presente ha tomado un interés creciente con el fin de evitar la pérdida de los conocimientos de las comunidades locales causada por las rápidas transformaciones sociales que ocurren

${ }^{1}$ Universidad Nacional de La Pampa, Facultad de Ciencias Exactas y Naturales. Ruta Nac. 35 km 334 (6300) Santa Rosa, La Pampa. E mail: wmuino@yahoo.com.ar 
(Alexiades 2003), pero también con el propósito de descubrir novedosas fuentes de compuestos químicos en las plantas (Prance \& al., 1994). En Argentina, las comunidades criollas han sido objeto de estudio particularmente en las últimas décadas, como dan testimonio diversos trabajos en la materia (Martínez Crovetto, 1981; Hilgert, 2001; Scarpa, 2002; Pochettino y Martínez, 2001; Muiño, 2010 b).

En la región que compete a este estudio las investigaciones referidas a la etnobotánica médica son escasas, dentro de la temática se pueden citar los estudios etnobotánicos sobre la población rural criolla (Muiño, 2010 a) y sobre la etimología de los nombres comunes y usos de las plantas en el Sur de Mendoza (Muiño \& al, 2006). También en el Sur mendocino, pero ya con un enfoque antropológico se puede mencionar el trabajo de Agüero Blanch (1968), que pone énfasis en el rol que cumplen los agentes sociales que participan en el proceso de curación de las dolencias, sus interpretaciones etiológicas y la farmacopea que utilizan en los procesos de curación. En el Norte mendocino, en las lagunas de Huanacache se destaca el trabajo de Montani y Vega Riveros (2010) con comunidades rurales actuales. También en el área de influencia del presente trabajo, merece una mención especial el estudio etnobotánico de Steibel (1997) que hace referencia a las plantas utilizadas por el pueblo ranquel en la provincia de La Pampa, en tanto que en la provincia de Córdoba existen varios trabajos en torno a la etnobotánica médica criolla (Arias Toledo, 2009; Trillo \& al, 2010, Martínez 2010) que establecen elementos de conexión con la medicina local de la región aquí tratada. Por otra parte en San Juan existen importantes aportes a la temática a través de los cuadernos de difusión publicados por Karlin \& al (2005, 2006).

No se puede dejar de mencionar la importancia que tienen como información de base las floras medicinales regionales relacionadas con el área de este estudio, tales son los trabajos de Del Vitto \& al (1997, 1998), Roig (2001), Barboza \& al (2006) y Steibel \& al (2008).

El trabajo que aquí se presenta toma relevancia por su contribución al conocimiento de las relaciones entre los humanos y las plantas considerando especialmente la necesidad de información etnobotánica en la región central de Argentina.

Junto con esa escasez de datos, las transformaciones que ocurren en la sociedad actual manifiestan una tendencia a la homogeneización cultural con un nivel de alcance geográfico extraordinario debido a los avances tecnológicos ocurridos principalmente en el área de las comunicaciones. En este contexto, los cambios en las formas de vida y el uso de los recursos naturales no escapan a estas influencias (Mateus \& Brasset, 2002; Bonasewicz, 2004). En función de ello, el objetivo de este trabajo consiste en caracterizar la interpretación del origen de las dolencias, registrar el conocimiento y uso actual de la farmacopea vegetal, así como la terapéutica aplicada en la práctica de la medicina de esta comunidad.

\section{Área de estudio}

El trabajo se realizó en el área que corresponde al ejido urbano de la localidad de La Humada, que abarca el sector occidental del departamento Chicalcó en el NW de la provincia de La Pampa. Está ubicado entre los $36^{\circ} 00^{\prime}$ y $36^{\circ} 47^{\prime}$ de latitud S y $67^{\circ} 40^{\prime}$ y $68^{\circ} 20^{\prime}$ de longitud W (mapa en Muiño 2010 b), limitando al $\mathrm{N}$ y al $\mathrm{W}$ con la provincia de Mendoza. Se ubica en la provincia fitogeográfica del Monte (Cabrera 1971) y el contexto florístico está caracterizado por una vegetación arbustiva, cuya especie dominante es Larrea divaricata Cav. acompañada por Prosopis flexuosa DC. var depressa F. A. Roig, con un estrato herbáceo de gramíneas y escasos árboles bajos de Prosopis flexuosa DC. f. Alexuosa, Condalia microphylla Cav. y Schinus johnstonii F. A. Barkley. Los habitantes de la región son en su mayoría colonos criollos de origen español asentados allí posteriormente a la campaña militar de 1879 que expulsó de sus dominios a la población aborigen. La actividad económica más importante es la cría extensiva de ganado vacuno y caprino. La población del sector rural profesa mayormente la religión católica, 
pero en la localidad de Chos Malal del Sur del departamento y en el área urbana de La Humada hay un mayor predominio del culto pentecostal. Una información más detallada sobre el proceso histórico de poblamiento y los aspectos etnográficos del área puede observarse en el estudio etnobotánico del autor (Muiño 2010 a).

\section{Materiales y Métodos}

La información etnobotánica se obtuvo durante nueve campañas entre 2005 y 2009. Se implementó la metodología clásica utilizada en los trabajos etnobotánicos que consiste en la obtención de los datos a partir de entrevistas realizadas con los pobladores y la documentación de su testimonio con especímenes vegetales identificados por ellos con sus nombres locales y sus atributos. De esta manera, la información fue registrada a lo largo de entrevistas abiertas y semiestructuradas a 27 mujeres y 47 varones adultos cuya edad promedio fue de 56 años. Las entrevistas abiertas permitieron identificar a los informantes más destacados por su conocimiento en el tema con quienes se trabajó posteriormente en mayor profundidad. Toda la información fue registrada en forma escrita y luego ordenada en una base de datos confeccionada mediante el programa informático Microsoft Office Access XP 2003 a partir del cual se llevó a cabo el análisis de la misma.

Para establecer la cantidad de entrevistas suficientes, se tuvo en cuenta la "Ley de rendimientos decrecientes" (Martin, 2000), no obstante se llevaron a cabo una cantidad adicional de las mismas con el fin de cubrir el área en forma más homogénea.

Todos los datos aportados por los entrevistados fueron tomados como válidos, aún aquellos que fueron mencionados solo una vez a lo largo de los encuentros. Cuando no pudo colectarse la planta o no pudieron obtenerse otras referencias de la misma, la información no fue considerada en el análisis. Por el contrario, las repeticiones de datos indicaron el amplio consenso que tienen algunas especies y dieron cuenta de la vigencia del uso o la importancia de algunas plantas en el pasado.

El material de referencia fue colectado en su mayoría con los entrevistados y en los casos en que su edad o su estado de salud lo impedían, la recolección se realizó previamente y luego se trabajó sobre la identificación e información de las especies en el domicilio del entrevistado, a la vista del material. Las recolecciones botánicas fueron interrumpidas en las campañas desarrolladas en otoño e invierno, no solamente porque son temporadas en que las plantas se encuentran en estado vegetativo sino porque gran parte de la vegetación de la zona es caducifolia.

Los ejemplares colectados fueron herborizados y depositados en el herbario de la Facultad de Agronomía de la Universidad Nacional de la Pampa (SRFA) y todos ellos fueron identificados taxonómicamente por el autor.

\section{Resultados}

\section{Etiología}

Las dolenciasregistradas en elárearesponden a etiologías variadas, pero la población local hace referencia a las enfermedades naturales y a las impuestas, caracterización que concuerda con las categorías émicas expresadas en los trabajos de Jiménez de Pupareli (1984) y García y Jiménez (1986) con campesinos del litoral argentino. De este modo aquí se hará referencia a los dos tipos de dolencias enunciadas. Entre las naturales se pueden ubicar las producidas por desequilibrios orgánicos o por la interacción entre el organismo y el ambiente. A estas pertenecen los "empachos", "pasmos", "aires", resfríos y otras. Por otra parte, las enfermedades impuestas se caracterizan porque en ellas el agente causal es una persona que ejerce un daño o mal sobre otra con o sin intencionalidad. A este grupo pertenecen las "ojeaduras", "daños" o males impuestos y la "istericia".

\section{Terapéutica}

El marco terapéutico encuentra sustento en 
procesos de oposición de antigua raigambre en la medicina, que se fundamentan en el concepto templario (Palma 1973). Esta teoría sostiene que la terapia consiste básicamente en restablecer el equilibrio perdido a través de la aplicación de una planta u otro elemento de naturaleza opuesta a la dolencia, que en estos casos es de carácter cálido o fresco. No obstante, este esquema explicativo se presenta en forma desarticulada e incompleta.

En la mayoría de las dolencias impuestas y en algunas de las naturales intervienen elementos y prácticas curativas que se relacionan con símbolos y rituales de la religión católica, esto se evidencia en la relevancia que adquieren el número tres o las oraciones que forman parte de las curas de palabra.

La farmacopea está constituida por un conjunto de componentes de origen mineral y biológico, estos últimos integrados por diversas partes de animales y sus productos metabólicos así como un amplio espectro de especies vegetales.

Las formas de administración varían de acuerdo al tipo de dolencia. En las inflamaciones se aplican lavajes, fricciones o paños embebidos en infusiones acuosas de plantas, cuya temperatura varía en función de la dolencia. En los casos que fue explicitado, las de origen cálido se tratan con preparados frescos y viceversa de acuerdo a los principios de oposición tal como se expuso anteriormente.

Las cataplasmas son otra variante de tratamiento externo, estas consisten en la aplicación directa de la planta triturada y macerada. No obstante, estas terapias son más específicas que las anteriores y se administran en contusiones y en fracturas de huesos.

Entre las terapias por vía oral se destacan las infusiones denominadas localmente como "té de" si se trata de una infusión caliente o "agua de" si la ingesta debe hacerse a temperatura ambiente.

\section{Los agentes sanitarios}

La práctica de la medicina local se lleva a cabo en el seno del hogar y los portadores de estos conocimientos son los adultos de ambos sexos. Su transmisión se realiza a partir de estos a los jóvenes, pero en la actualidad se observan discontinuidades en esta práctica debido a la importante presencia de los programas de asistencia sanitaria gubernamental y a la influencia del discurso de la medicina oficial que con frecuencia confronta con la práctica médica tradicional.

En cuanto a las terapias de carácter religioso, ya se destinen estas al tratamiento de dolencias naturales o sobrenaturales, las curas son llevadas a cabo a través de especialistas y yuyeros que conocen el tratamiento de estos trastornos específicos. La eficacia de estos tratamientos se considera dependiente tanto de la potencia sanadora del especialista como de la fe religiosa del paciente.

\section{Farmacopea vegetal}

La recolección de plantas se realiza en cualquier época del año según las necesidades y en general, la costumbre es hacer la colecta en el momento de uso. Sin embargo, con las especies anuales y las caducifolias suele efectuarse en primavera y verano. Si la colecta de las últimas se efectúa cuando las hojas han caído, la materia prima empleada son las raspaduras de los tallos eliminando previamente el súber. Este producto se denomina localmente "entrecáscara" y es utilizado en infusiones. Cuando se trata de plantas cultivadas se indicó que la recolección debe efectuarla su propietario, es decir, la persona que en el predio se dedica a su cultivo. Se señaló también que esta actividad debe ser evitada por las mujeres durante el período menstrual. No obstante, esta costumbre no se adopta en forma generalizada.

El intercambio de especies cultivadas con gente de pueblos aledaños de Mendoza es muy fluido. Esto se destaca también para las especies silvestres no disponibles en el área y propias de ambientes serranos, más comunes en esa provincia.

Las partes de las plantas utilizadas varían de acuerdo a la dolencia. En los tratamientos intervienen en orden de importancia las partes vegetativas aéreas, las raíces, rizomas y bulbos. En menor proporción lo hacen las flores, frutos y semillas.

Se registró un total de 90 taxones específicos 
e infraespecíficos de uso medicinal dentro de los cuales 57 son silvestres recolectados, 15 cultivados y 18 adquiridos comercialmente o intercambiados con vecinos y familiares de otras áreas. El conjunto está representado por 42 familias botánicas. De ellas, Asteraceae es la que posee mayor cantidad de taxones en uso (28\%), seguida por Verbenaceae $(7.8 \%)$, Lamiaceae $(5,6 \%)$ y Solanaceae $(4.4 \%)$. La especie con mayor diversidad de usos es $L$. divaricata ("jarilla") siendo asimismo el taxón con mayor cantidad de citas.

En cuanto al origen de los taxones específicos e infraespecíficos, se registró el empleo de 33 adventicios y 57 nativos. De estos últimos, 25 son endemismos de Argentina. (URL:http// darwin.edu.ar/Proyectos/FloraArgentina/ especies.asp)

Debido a la amplitud de los datos y a fin de facilitar el ordenamiento y la lectura de la información referida a este punto, las plantas empleadas en la medicina tradicional se presentan en orden alfabético en la Tabla 1 que aparece como anexo. Se consideró conveniente incluir aquí también las especies saponificables y fragantes empleadas en el aseo personal considerando su estrecha vinculación con la salud. En el catálogo se consignan además los nombres científicos y comunes, la familia de pertenencia, el número de colección y la información referida a su significado y uso.

\section{Discusión}

La interpretación del origen de las dolencias por la población es similar a la registrada en otras áreas rurales de Argentina (Jiménez de Pupareli 1984, García y Jiménez 1986). Algo similar ocurre con sus denominaciones que en ocasiones pueden tener ligeras diferencias según la región. Es así como la "istericia" aquí mencionada coincide plenamente con el origen y sintomatología que describe Idoyaga Molina para el NOA bajo la denominación de "tirisia" o "tisiadura", definida como: "...una afección específica que se hace evidente en la inapetencia, el dolor de cabeza, el llanto, la tristeza por la pérdida de vínculos sociales" (Idoyaga Molina 2001 a: 11).
Las prácticas curativas se sustentan en modelos terapéuticos de oposición en correspondencia con el síndrome cálidofresco. De este modo las características de la dolencia y la terapia confluyen en este contexto racional vinculado con la teoría hipocrática, fenómeno que ya fue observado en diferentes comunidades rurales de Argentina (Scarpa, 2002; 2004; Martínez, 2010; Trillo \& al., 2010) y de Hispanoamérica (Foster 1953). $\mathrm{Si}$ bien existen elementos comunes a estas comunidades en lo que respecta al modelo terapéutico, también se observan marcados contrastes, en especial con el marco etiológico, como lo demuestra el hecho de que aquí no se registraron dolencias de manera explícita cuyo origen tuviera connotaciones de carácter cálido o fresco. Estas solamente se pudieron deducir a partir de la caracterización de un escaso número de plantas a las cuales se les atribuye la propiedad de cálidas y de la racionalidad en la que se fundamentan las propuestas terapéuticas. En este sentido, la estructura del modelo de la medicina tradicional adquiere un carácter fragmentario con evidentes procesos de erosión. Es probable que las causas que dan origen a esta situación estén vinculadas al discurso hegemónico que ejerce la medicina oficial, en general hostil a las prácticas médicas tradicionales, y a una destacada asistencia sanitaria por parte del estado provincial a través de infraestructura, insumos gratuitos y profesionales de la materia.

Un fenómeno similar ocurre con la presencia de símbolos y rituales del catolicismo en la terapéutica local, ya que aquí tienen menor representatividad, si se la compara con la que adquieren en la medicina local de otras comunidades criollas del NOA y Cuyo (Hilgert 2001; Idoyaga Molina 2001 a; Idoyaga Molina 2001 b). Esta situación puede deberse a que la región posee una presencia comparativamente menos importante de la Iglesia Católica, y a un sostenido avance en las últimas décadas de las Iglesias Pentecostales, no obstante este análisis escapa a los fines de este trabajo pero puede ser base para futuras investigaciones. Junto a las similitudes enunciadas, también se pueden verificar las mismas pautas preventivas registradas en las culturas del Norte argentino en relación con la recolección de las plantas 


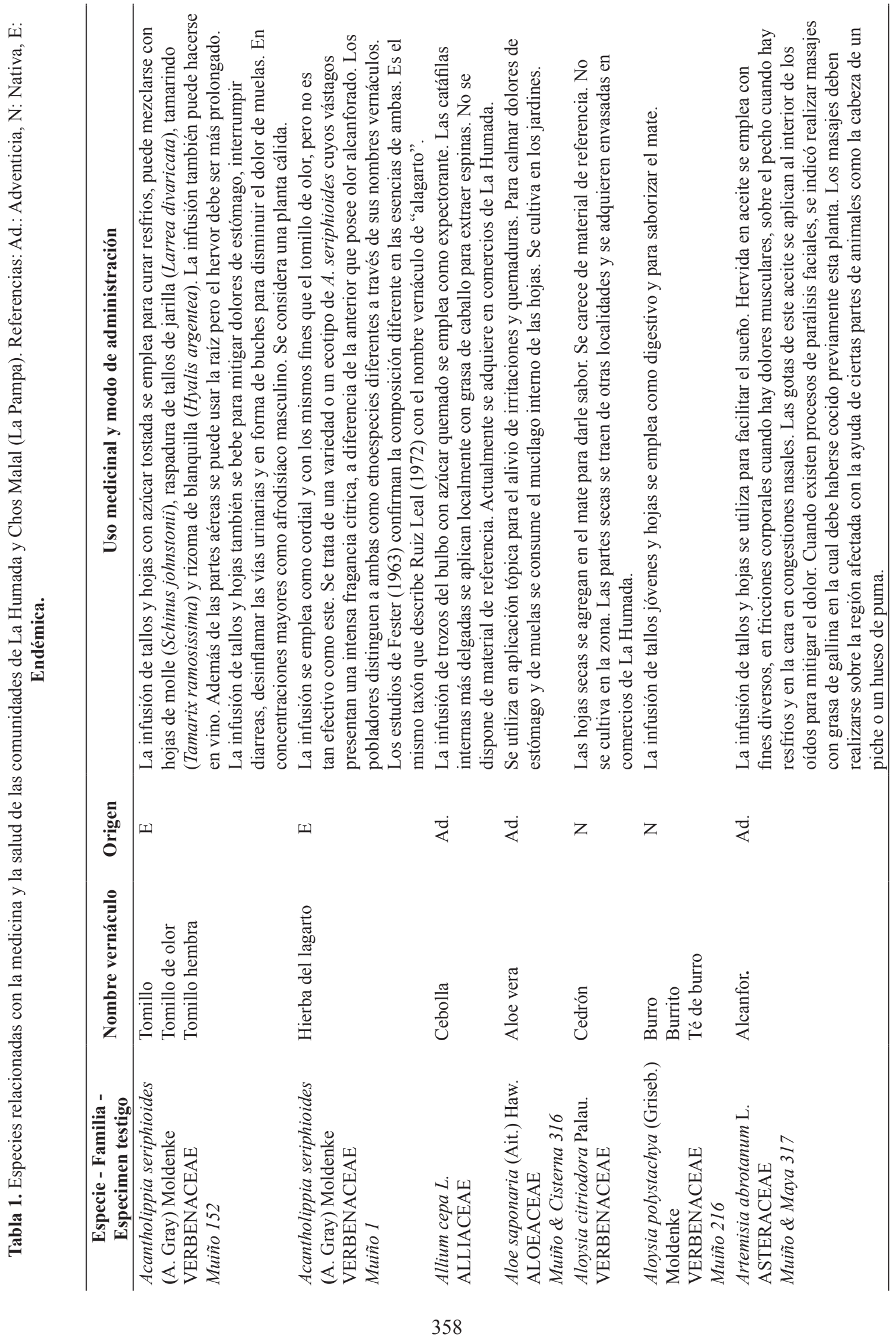




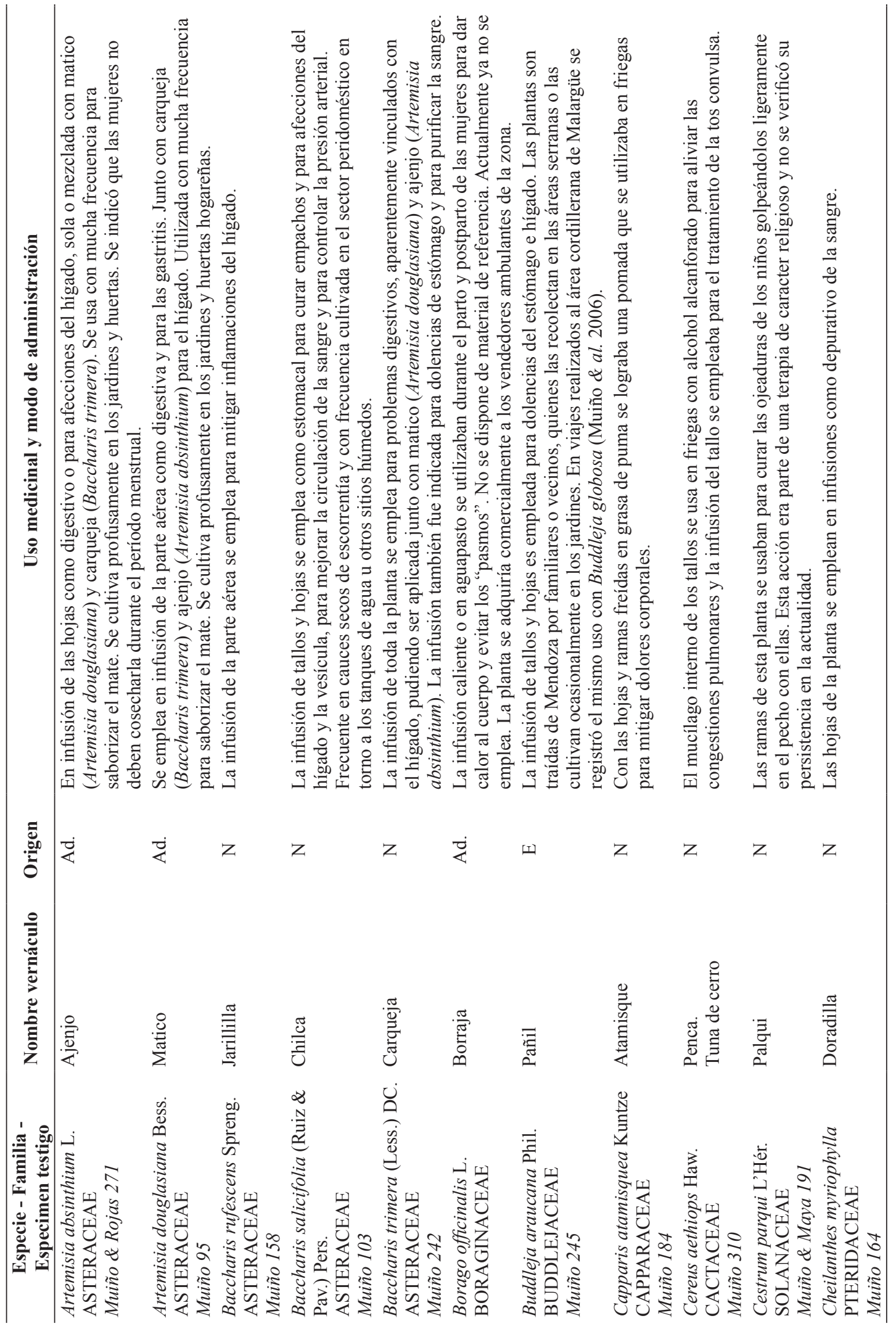




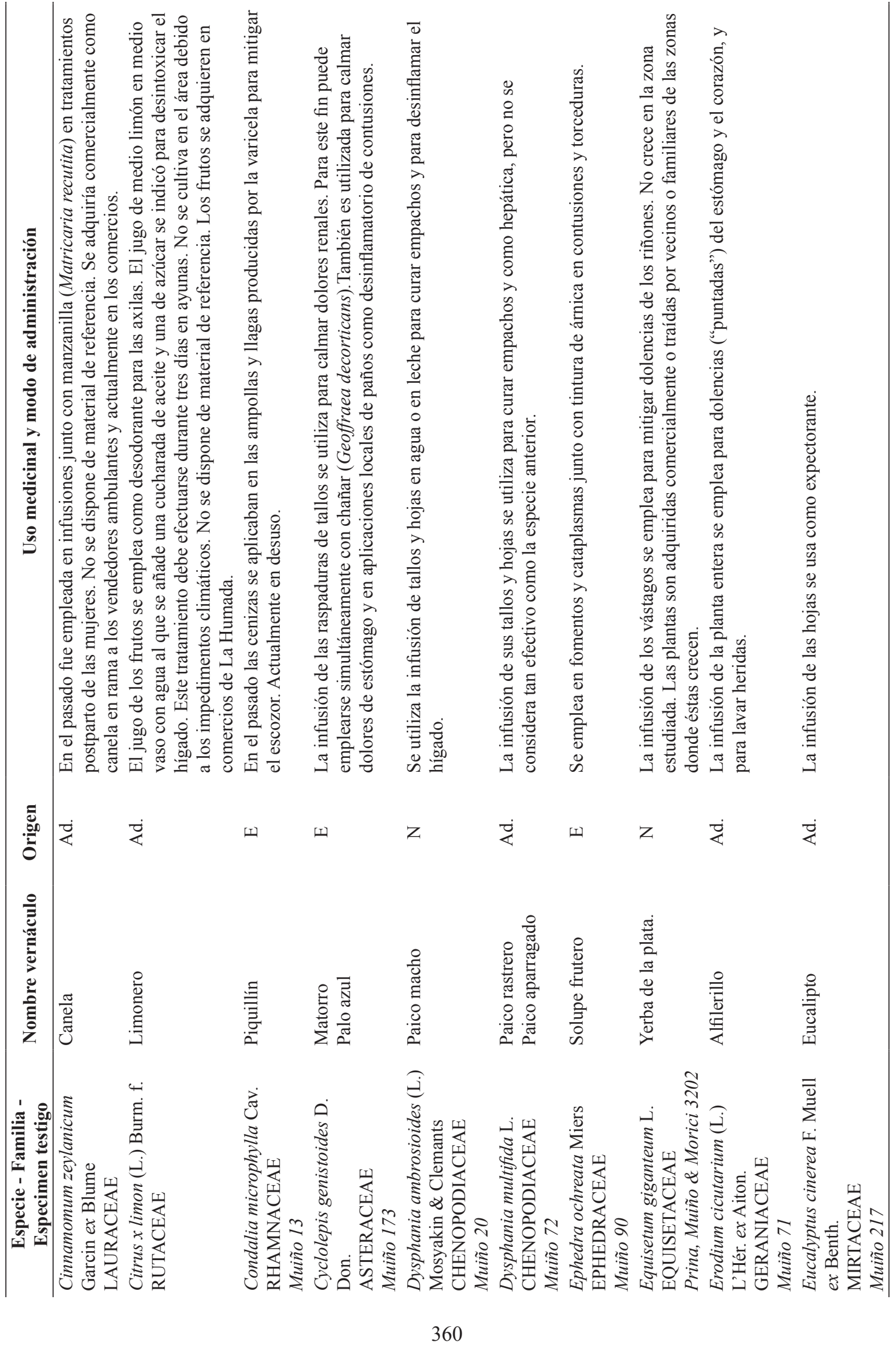




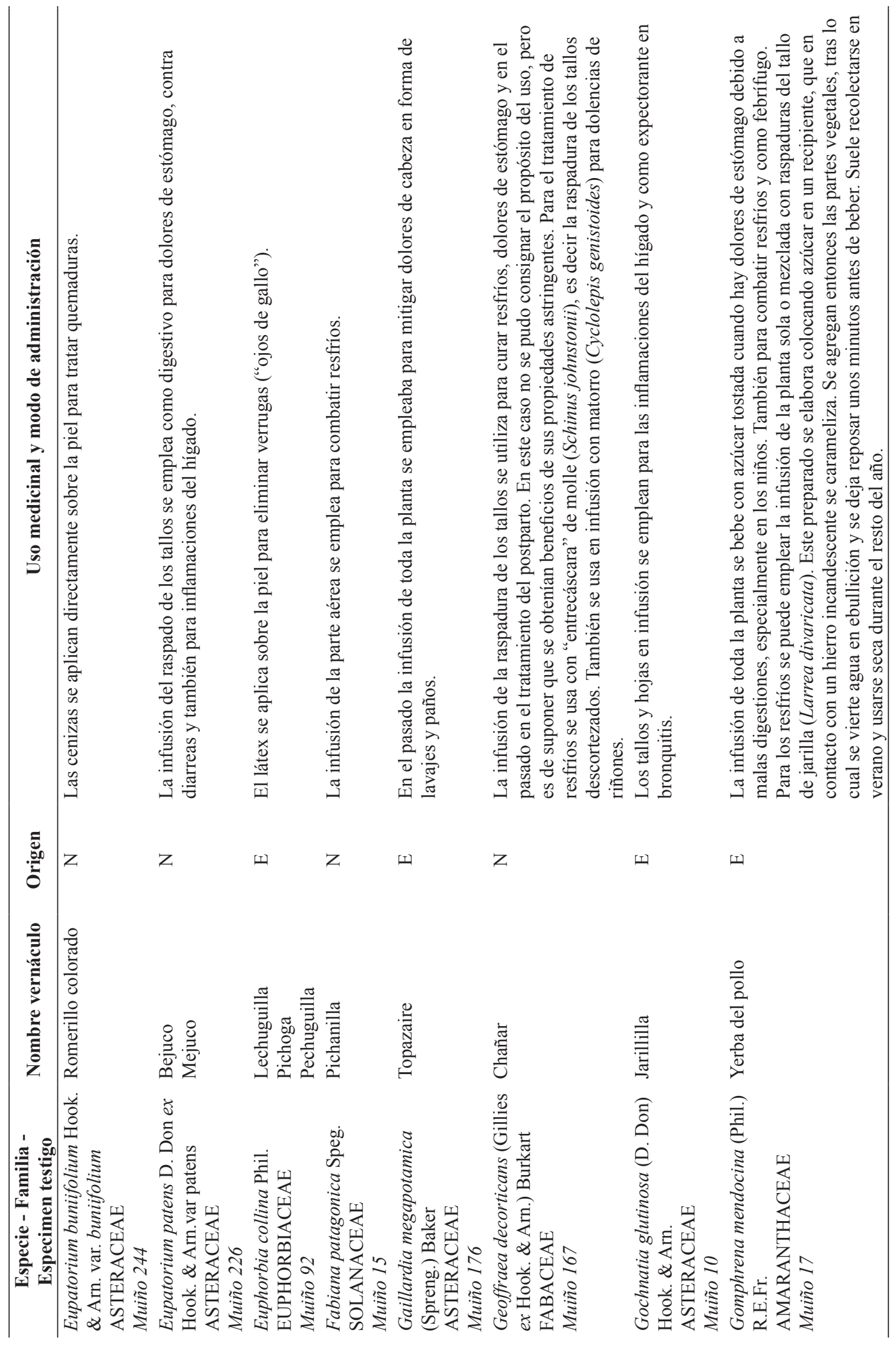


BONPLANDIA 20(2). 2011

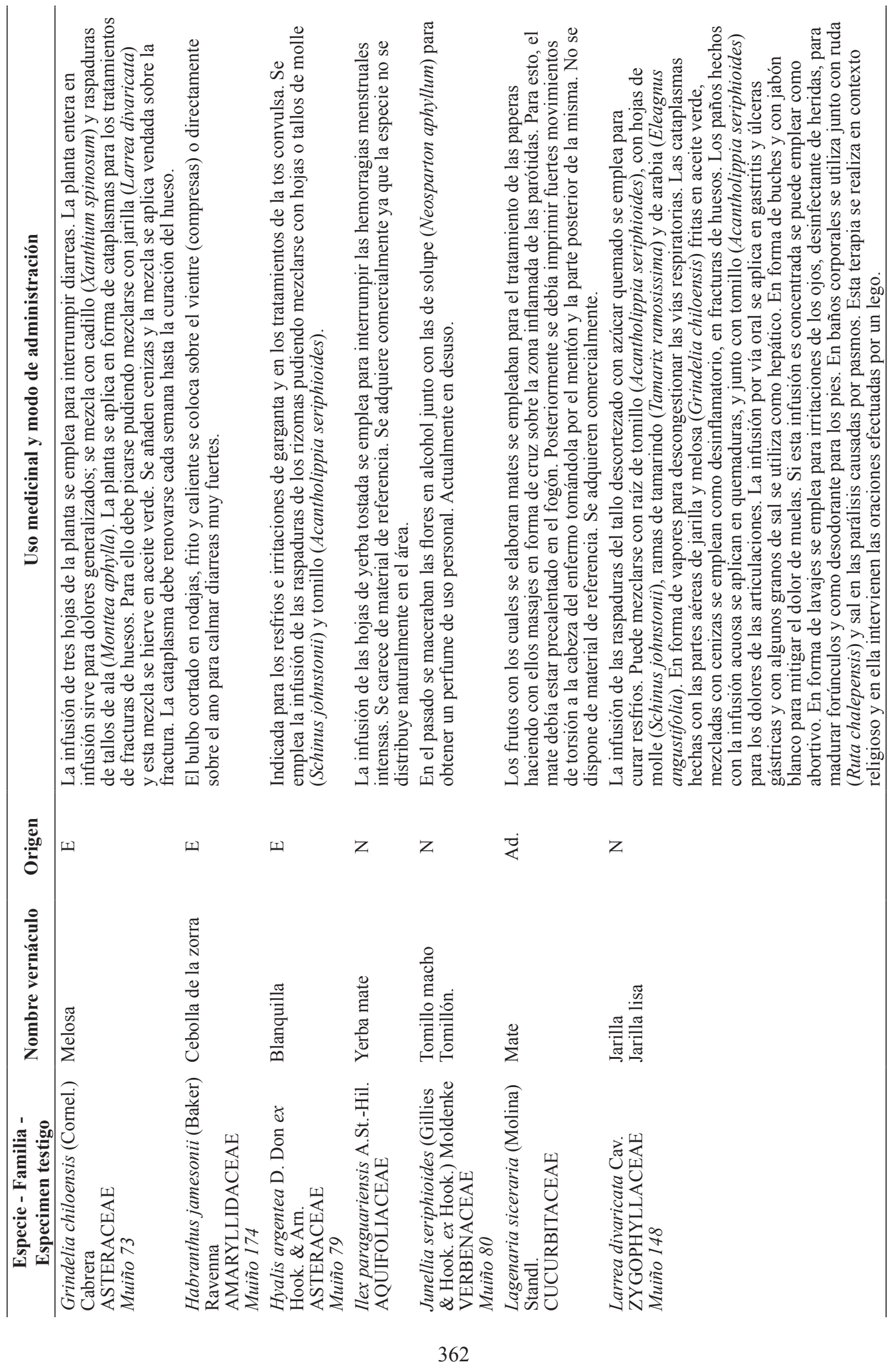




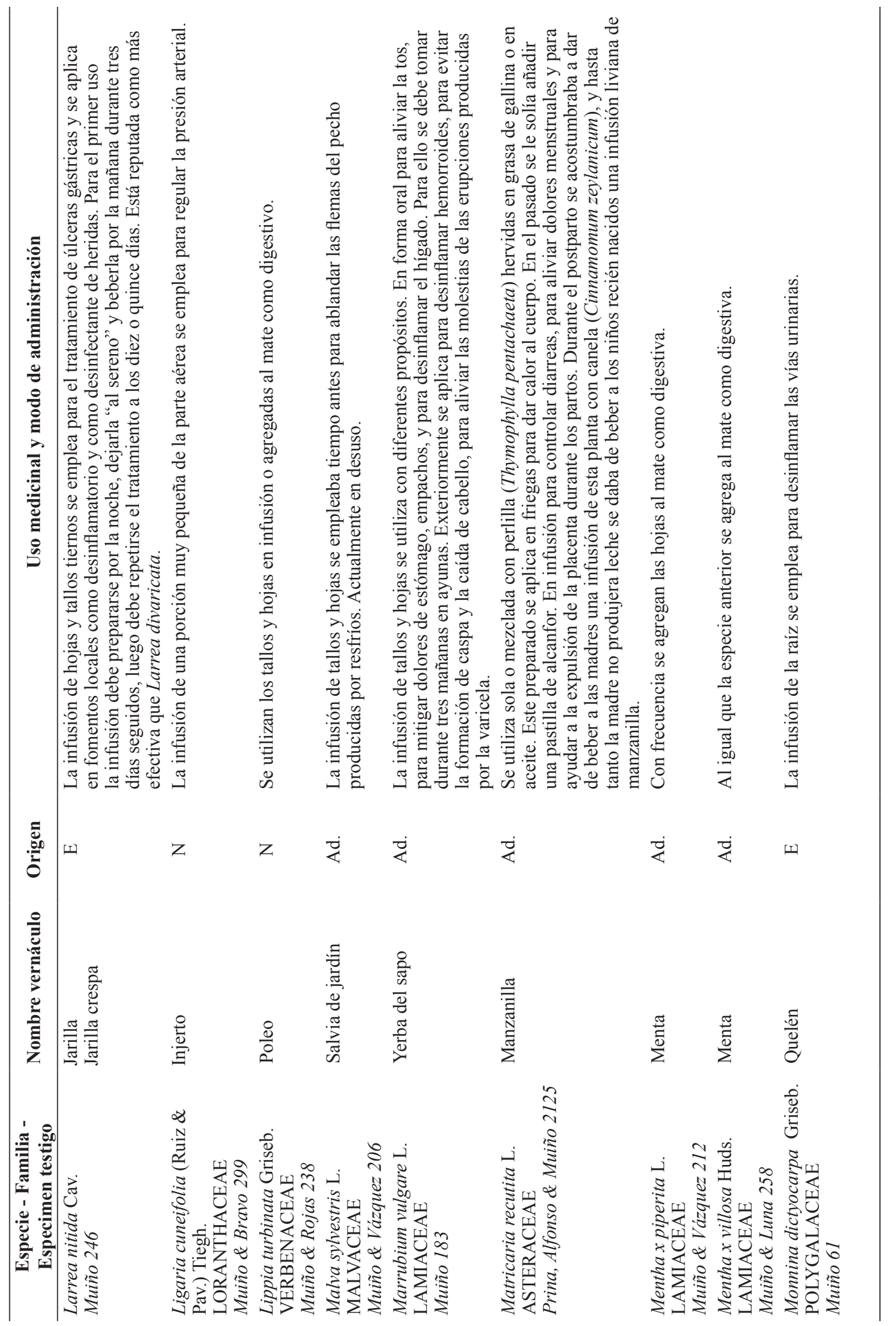


BONPLANDIA 20(2). 2011

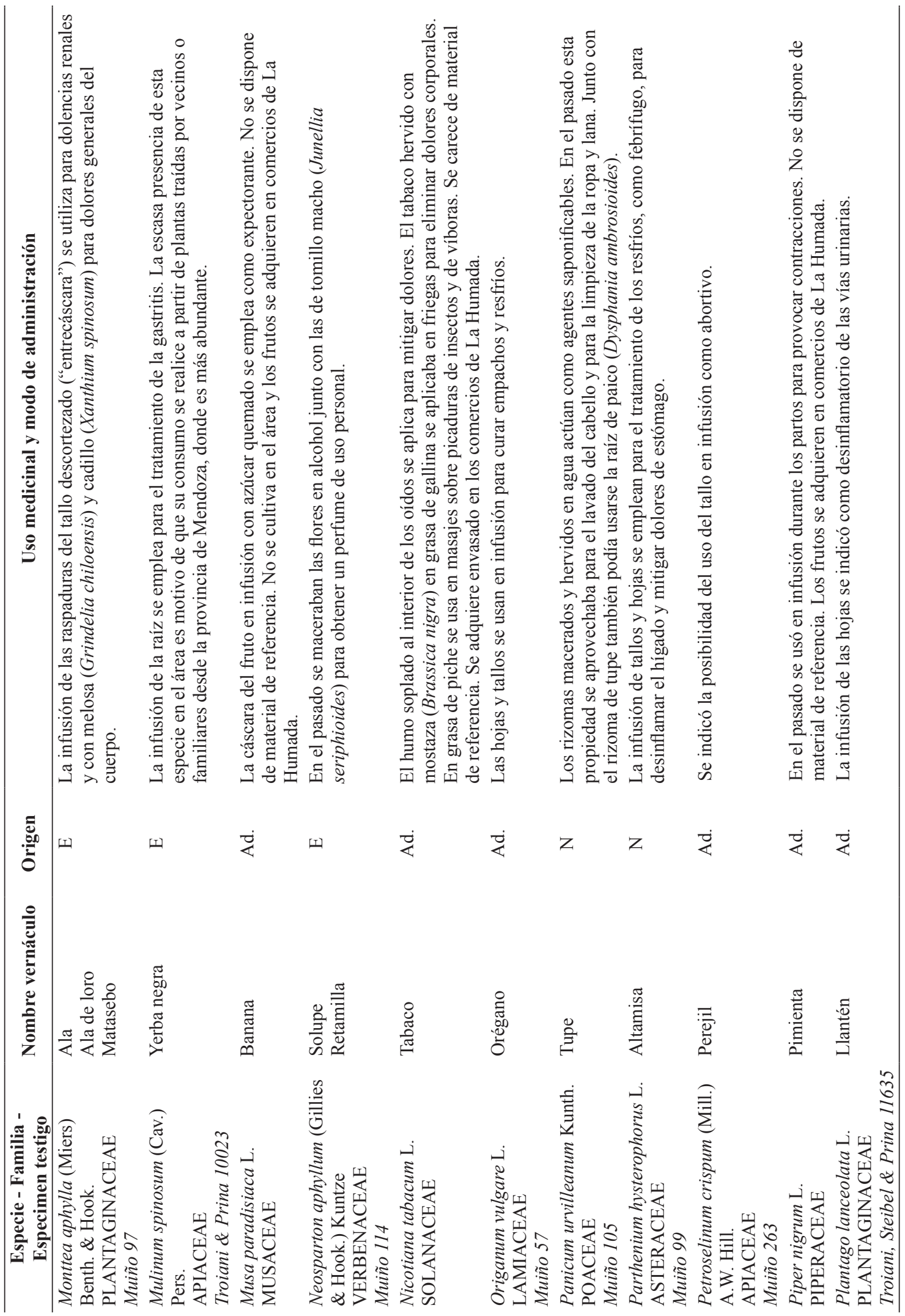




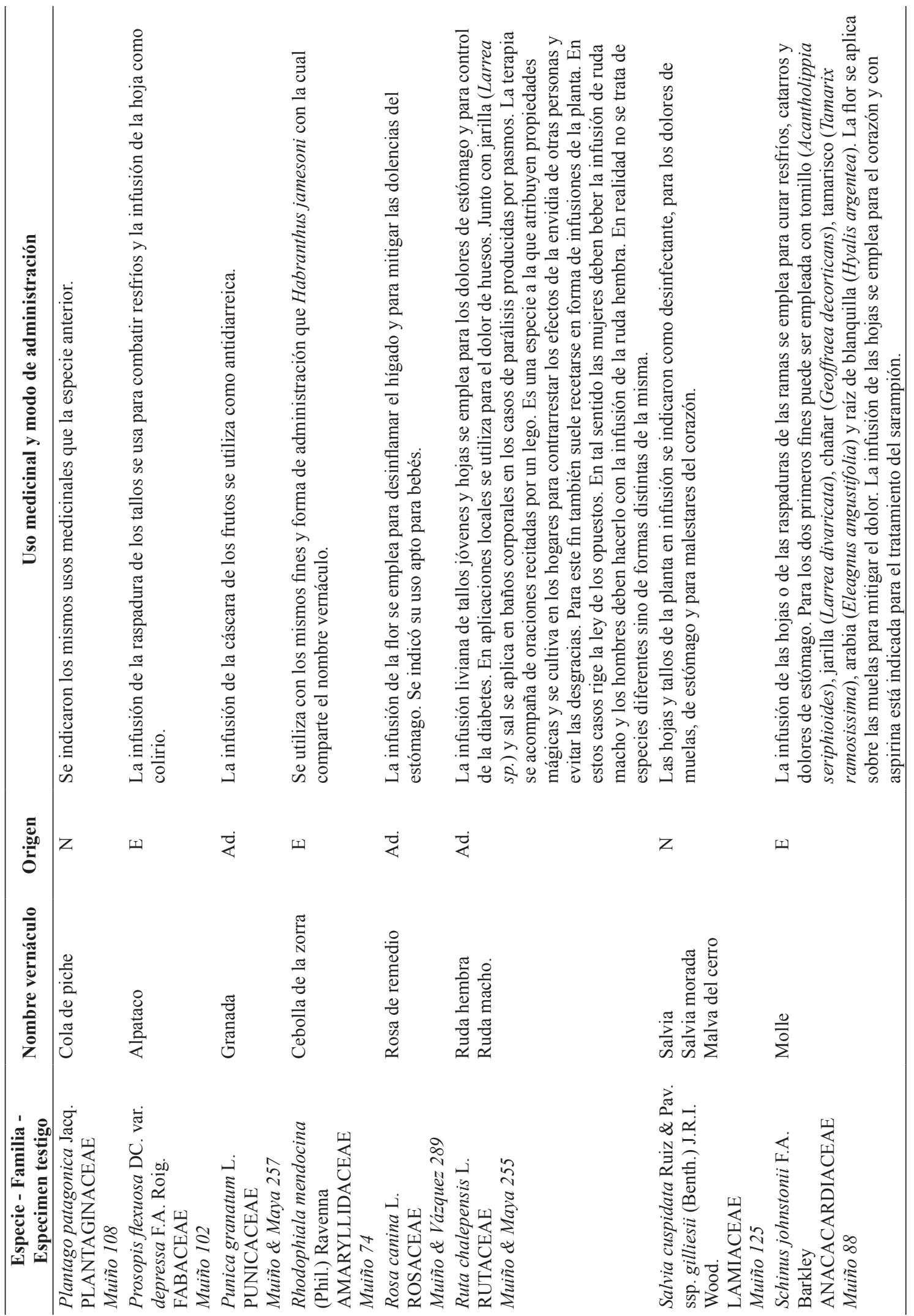




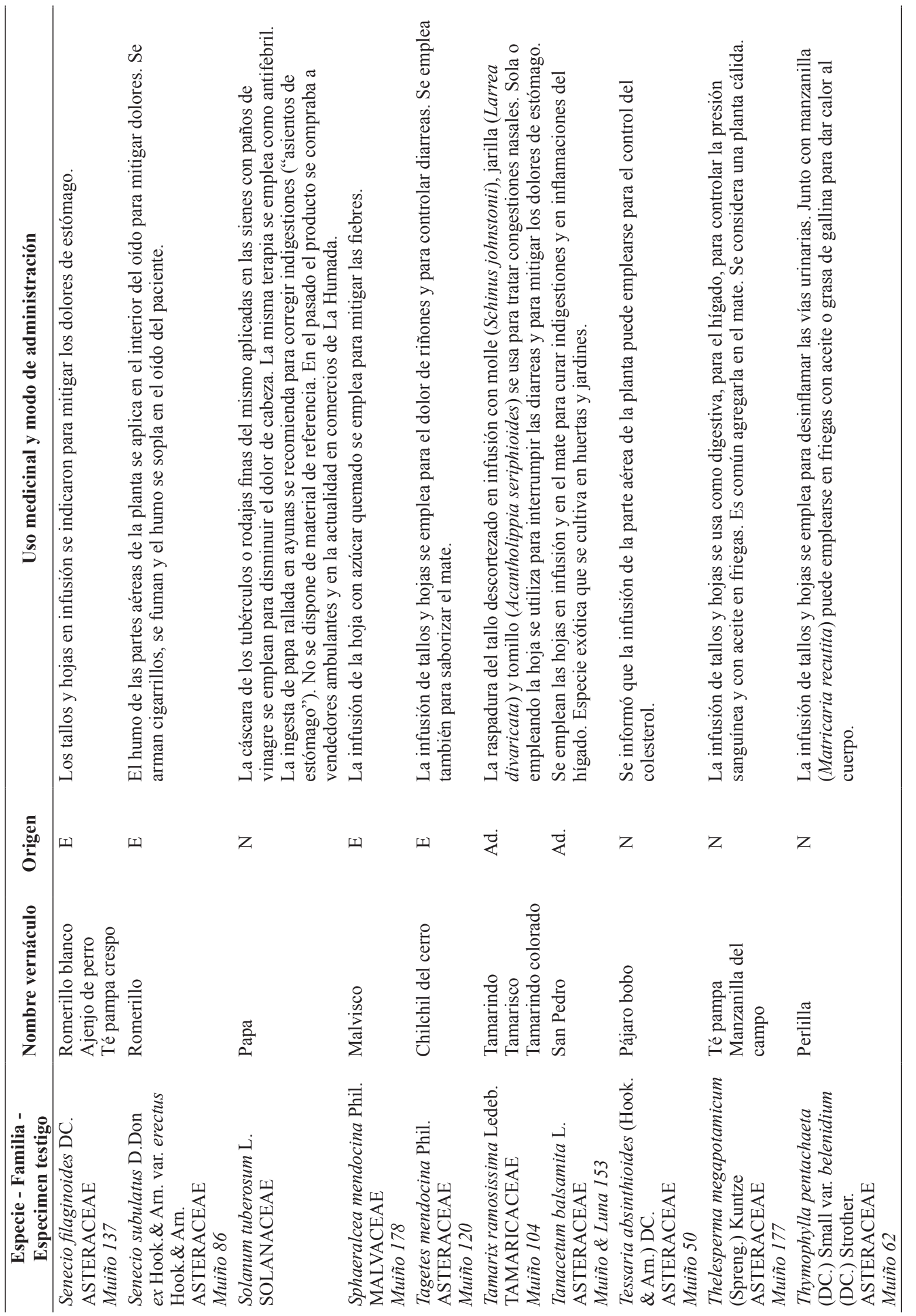


W. A. Muiño, La etnobotánica médica del área de transición pampeano cuyana

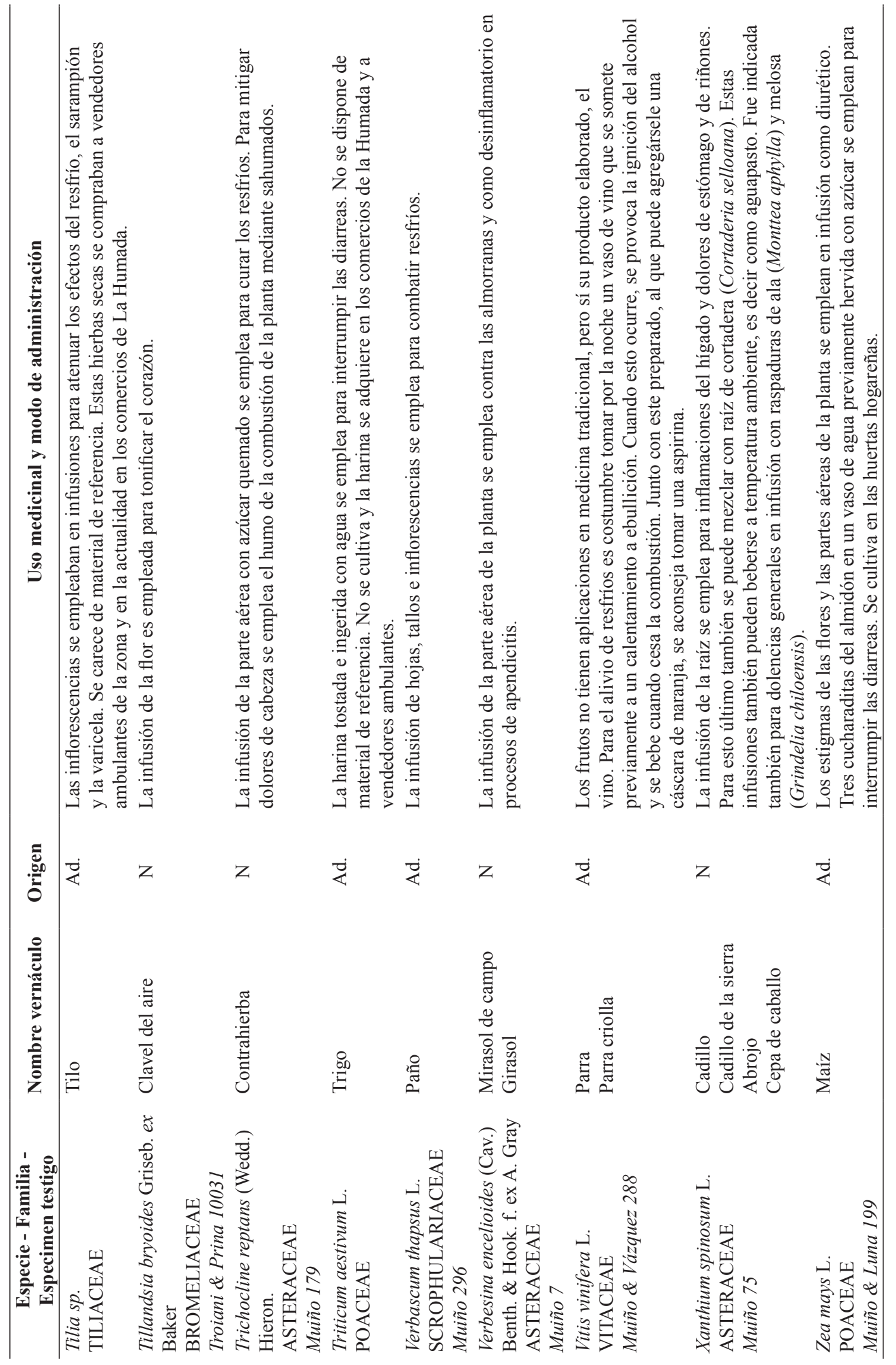


cultivadas (Arenas y Galafassi, 1994).

La diversidad florística de la región es relativamente baja si se compara con otras regiones fitogeográficas de Argentina (Cabrera, 1971), sin embargo, del total de especies registradas, la mayor parte son silvestres y dentro de este grupo, la mayor diversidad de usos corresponde a las más abundantes, como L. divaricata, Acantholippia seriphioides (A. Gray) Moldenke o S. johnstonii. Si bien no se puede establecer como regla general que la disponibilidad es el único factor de influencia en el uso preferencial de algunas especies (Akerreta \& al, 2007), en regiones con estas características, donde el clima promueve una uniformidad en la vegetación y la dominancia de determinados taxones, su facilidad de acceso estaría actuando como un condicionante de uso importante. Sin embargo también existen otros, ausentes o escasos en la zona, como Buddleja araucana Phil. y Mulinum spinosum (Cav.) Pers., que debido a su comprobada efectividad los pobladores no dudan en buscar su aprovisionamiento en localidades mendocinas aún existiendo otras especies en el área que pueden cumplir la misma función.

\section{Conclusión}

En el área estudiada, los modelos etiológico y terapéutico de la medicina local son congruentes con los observados en otras áreas rurales del centro y norte de Argentina. No obstante, aquí se presentan desarticulados e incompletos, con claros signos de erosión. Los símbolos y rituales del catolicismo marcan un a impronta destacada en las terapias, pero también aquí su presencia es menor comparada con la medicina tradicional del Norte argentino y de Cuyo.

En el conjunto de especies empleadas en la medicina local, se destaca un importante número de especies silvestres recolectadas (63\%), y entre ellas, las más abundantes son las que presentan la mayor diversidad de usos. Esta diversificación está relacionada con trastornos de origen respiratorio digestivo, urinario y traumatológico.

Los procesos de erosión verificados en este estudio en torno al empleo de la herbolaria médica pone una vez más el acento en la importancia del registro de los conocimientos y usos botánicos actuales e históricos de cada región.

\section{Agradecimientos}

Deseo expresar mi agradecimiento al Licenciado Pastor Arenas Rodríguez por la lectura crítica del manuscrito y a los integrantes de las comunidades de La Humada y Chos Malal que participaron en las entrevistas. Este trabajo fue financiado por la Facultad de Ciencias Exactas y Naturales de la Universidad Nacional de La Pampa.

\section{Bibliografía}

AGÜERO BLANCH, V. O. 1968. Las remedieras de Malargüe. Instituto de Antropología. Universidad Nacional de Córdoba. Córdoba. 34 pp.

AKERRETA, S; R. Y. CAVERO; V. LÓPEZ \& M. I. CALVO. 2007. Analyzing factors that influence the folk use and phytonomy of 18 medicinal plants in Navarra. JEE 3: 16. doi:10.1186/1746-4269-3-16

ALEXIADES, M. N. 2003. Ethnobotany in the Third Millenium: expectations and unresolved issues. Delpinoa 45: 15-28.

ARENAS P. \& G. P. GALAFASSI. 1994. La ruda (Ruta chalepensis L.-Rutaceae-) en la medicina folklórica del Norte argentino. Dominguezia 11 (1):32.

ARIAS TOLEDO, B. 2009. Diversidad de usos, prácticas de recolección y diferencias según género y edad en el uso de plantas medicinales en Córdoba, Argentina. BLACPMA 8 (5): 389-401.

BARBOZA, G. E., J. J. CANTERO, C. O. NÚÑEZ Y L. ARIZA ESPINAR (EDS.). Flora medicinal de la provincia de Córdoba (Argentina). Pteridófitas y Antofitas silvestres o naturalizadas. Museo Botánico. Córdoba. 1250 pp.

BONASEWICZ, A. 2004. Las causas y los efectos de la globalización. Actas Latinoamer. Varsovia 27: 39-44.

CABRERA, A. L. 1971. Fitogeografía de la República Argentina. Bol. Soc. Argen. Bot. 14 (1-2).

DEL ViTTO, L. A., E. M. PETENATTI \& M. E. PETENATTI. 1997. Recursos herbolarios de San Luis (República Argentina). Primera parte: plantas nativas. Multequina 6: 49-66.

. 1998. Recursos herbolarios de San Luis (Argentina). Segunda parte: plantas exóticas cultivadas, 
adventicias y/o naturalizadas. Multequina 7: 29-48.

FESTER, G. A. 1963. Variedades fitoquímicas en plantas aromáticas argentinas. Boletín de la Sociedad Argentina para la Investigación de Productos Aromáticos 5: 1-5.

FOSTER, G. 1953. Relationships between Spanish folk medicine and Spanish-American folk medicine. $J$. Am. Folk. 66: 201-217.

GARCÍA, S. P. \& D. I. JIMÉNEZ. 1986. Natural y postizo: Frío y caliente. Sistemas clasificatorios vigentes entre criollos del litoral argentino. Supl. Antropol. 21 (1): 131-146.

HILGERT, N. I. 2001. Plants used in home medicine in the Zenta River basin, Northwest Argentina. $J$. Ethnopharmacol. 76: 11-34.

IDOYAGA MOLINA, A. 2001 a. Etiologías, síntoma y eficacia terapéutica. El proceso diagnóstico de la enfermedad en el Noroeste argentino y Cuyo. Mitológicas 16: 9-43.

_ 2001 b. Lo sagrado en las terapias de las medicinas tradicionales del NOA y Cuyo. Scr. Ethnol. 23: 9-75.

JIMÉNEZ DE PUPARELI, D. 1984. Función de la medicina popular en la comunidad entrerriana y su relación con la medicina oficial. In Fundación F. G. Bracht (ed.), Cultura tradicional del área del Paraná Medio, pp. 237-254. Instituto Nacional de Antropología, Buenos Aires.

KARLIN, U., M. MARTINELLI, M. GAVIORNO, G. DÍAZ, Y C. ORDÓÑEZ. 2005. Saberes que sanan. Plantas nativas con uso medicinal en Balde del Rosario. San Juan, Argentina. Universidad Nacional de San Juan. 50 pp.

KARLIN, U.O., M. MARTINELLI, O. DAMIANI, G. DÍAZ, C. ORDÓÑEZ, M. GAVIORNO, C. MEGLIOLI, M. OJEDA, R. COIRINI, Y. RIBAS, M. HADAD, V. ESCOBAR. 2006. Huellas de identidad. Uso y conservación de las plantas en Tudcum, Malimán y Angualasto. Universidad Nacional de San Juan. 79 pp.

MARTIN, G. J. 2000. Etnobotánica. Manual de Métodos. Nordan - Comunidad. Montevideo. Uruguay. 240 pp.

MARTÍNEZ, G. J. 2010. Las plantas en la medicina tradicional de las sierras de Códoba. Un recorrido por la cultura campesina de Paravachasca y Calamuchita. Ediciones del Copista. 210 pp.

MARTÍNEZ CROVETTO, R. 1981. Plantas reguladoras de la fecundidad utilizadas en la medicina popular del Nordeste argentino. Parodiana 1 (1): 97-118.

MATEUS, J. R. \& D. W. BRASSET. 2002. La globalización: sus efectos y bondades. Econ. Desarro. 1 (1): 65-77.

MONTANI, M.C. Y C. VEGA RIVEROS (EDS.).
2010. Uso medicinal de plantas en la comunidad de Lagunas del Rosario, Mendoza, Argentina. Universidad Nacional de San Juan. 97 pp.

MUIÑO, W. A. 2010 a. El uso de las plantas Silvestres por la Comunidad de Chos Malal (provincia de La Pampa). Tesis para acceder al grado de Doctor en Ciencias Naturales. Facultad de Ciencias Naturales y Museo. Universidad Nacional de La Plata. La Plata.

- 2010 b. Ethnobotanical study of the rural population of the West of the Pampa Plain (Argentina). ERA 8:219-231.

—, G. L. ALFONSO \& A. O. PRINA. 2006. Origen de los nombres vernáculos y usos de las plantas por parte de los pobladores del Sur mendocino. Encuentro de Lenguas Indigenas Americanas, Santa Rosa, La Pampa, 15 al 17 de junio de 2006. 9 pp.

PALMA, N. H. 1973. Estudio antropológico de la medicina popular de la Puna argentina. Ed. Cabargon. Bs As. 131 pp.

POCHETTINO, M. L. \& M. R. MARTÍNEZ. 2001. Aporte al conocimiento actual de las plantas medicinales en Argentina. Estudio etnobotánico en el Departamento de Molinos, Provincia Salta, Argentina. In A.G. Amat (ed.), Farmacobotánica y Farmacognosia en Argentina (1980-1999), pp. 5586. Ediciones Científicas Americanas, La Plata.

PRANCE, G. T.; D.J. CHADWICK; J. MARSH (EDS.). 1994. Ethnobotany and the search for new drugs. New York, John Wiley \& Sons.

ROIG, F. A. 2001. Flora Medicinal Mendocina. Las Plantas Medicinales y Aromáticas de la Provincia de Mendoza (Argentina). EDIUNC. Mendoza. 305 pp.

RUIZ LEAL, A. 1972. Flora Popular Mendocina. Deserta 3: 3-296.

SCARPA, G. F. 2002. Plantas empleadas contra trastornos digestivos en la medicina tradicional criolla del Chaco Noroccidental. Dominguezia 18: 36-50.

—. 2004. El síndrome cálido-fresco en la medicina popular criolla del Chaco argentino. Rev. Dialectolog. Tradic. Pop. 59 (2): 5-29.

STEIBEL, P. E. 1997. Nombres y usos de las plantas aplicados por los indios Ranqueles de La Pampa (Argentina). Rev. Fac. Agronomía - UNLPam 9 (2): $1-40$.

STEIBEL, P., R. TOSO Y H. TROIANI. 2008. Plantas medicinales de la región pampeana. Fundación Allignani. Santa Fé. 264 pp.

TRILLO, C.; B. ARIAS TOLEDO; L. GALETTO \& S. COLANTONIO. 2010. Persistence of the Use of Medicinal Plants in Rural Communities of the Western Arid Chaco [Córdoba, Argentina]. OCMJ 2: 80-89.

URL:http//darwin.edu.ar/Proyectos/FloraArgentina/ especies.asp (consulta 10/12/11).

Original recibido el 1 de julio de 2011; aceptado el 12 de diciembre de 2011. 
